\title{
Spectral Imaging and Multivariate Statistical Analysis from Thin Specimens in the SEM with a Four-Channel Silicon Drift Detector
}

\author{
P.G. Kotula and J.R. Michael
}

Sandia National Laboratories, PO Box 5800, Albuquerque, NM 87185-0886

Silicon drift detectors represent a breakthrough in energy-dispersive x-ray analysis by allowing acquisition of spectral images at high count-rates while maintaining good spectral resolution. Additionally, newly available Si-drift detector arrays [1] offer large solid angle of collection allowing thin specimens to be analyzed in STEM in SEM mode. In this paper we describe the first results from Sandia's new Roentec four-detector Si-drift x-ray detector array. In particular we describe large spectral images acquired in less than five minutes from thin specimens. All of the data gave been analyzed with Sandia's Automated eXpert Spectral Image Analysis (AXSIA) multivariate statistical analysis software [2].

A $\mathrm{ZnO}-\mathrm{Bi}$ varistor specimen was prepared by FIB to a thickness of $100 \mathrm{~nm}$ and extracted ex situ and placed upon a carbon-coated $\mathrm{Cu}$-grid. The grid was then placed over a $2 \mathrm{~mm}$ hole drilled half-way into a graphite block. The specimen was then imaged in a Zeiss Supra 55 operated at $5 \mathrm{kV}$ and 30 $\mathrm{kV}$ with probe currents of $7 \mathrm{nA}$ and $14 \mathrm{nA}$ respectively with comparable probe sizes. For comparison, the same specimen was imaged in a FEI Tecnai F-30ST operated at 300kV in STEM mode with an EDAX SUTW Si (Li) EDS. In the SEM, the drift detectors were operated at the 220 ns time constant resulting in count-rates of 60-80 kcps stored. Spectral images consisting of $512 \times 384$ $(\sim 200,000)$ pixels by 500 channels at $5 \mathrm{kV}$ and 1000 channels at $30 \mathrm{kV}$, were acquired in 200 seconds resulting in $\sim 8 \mathrm{M}$ counts or 40 counts/spectrum at $5 \mathrm{kV}$ and $\sim 14 \mathrm{M}$ counts or $\sim 70$ counts/spectrum at $30 \mathrm{kV}$. AXSIA was used to analyze the data as previously described [2] via multivariate curve resolution. The data were $2 \mathrm{x}$ spatially compressed prior to analysis but the results were upsampled back to the original image size prior to output [3]. Figures 1-3 shows the $\mathrm{Bi}$ component image and spectral shape from the spectral image acquired at $5 \mathrm{kV}, 30$ and $300 \mathrm{kV}$ respectively. In addition to clusters of Bi-oxide, Bi has decorated the grain boundaries. This is evident from Figs. 2 and 3 but not Fig. 1. From Fig. $3(\sim 23 \mathrm{~nm} /$ pixel) the grain boundary segregation is less than two pixels wide. A comparable profile, although with a higher background, was observed at $30 \mathrm{kV}$. At $5 \mathrm{kV}$ no grain boundary segregation was visible[4].

References

[1] T. Schülein, R. Terborg, M. Rohde, Microsc Microanal 11(Suppl 2) (2005) 460.

[2] P.G. Kotula, M.R. Keenan, and J.R. Michael, Microsc. Microanal. 9 (2003) 1-17.

[3] P.G. Kotula and M.R. Keenan, Microsc. Microanal. 9 (Suppl 2) (2003) 152.

[4] The authors would like to acknowledge the assistance of Bonnie McKenzie with the acquisition of spectral images, Gary Bryant for fabrication of the graphite specimen holders, David Melgaard for the provision of the data translation software and Martin Rohde of Roentec Gmbh for providing the spectral image file format. Sandia is a multiprogram laboratory operated by Sandia Corporation, a Lockheed Martin Company, for the United States National Nuclear Security Administration, which is part of the Department of Energy (DOE), under contract DE-AC04 94AL85000. 

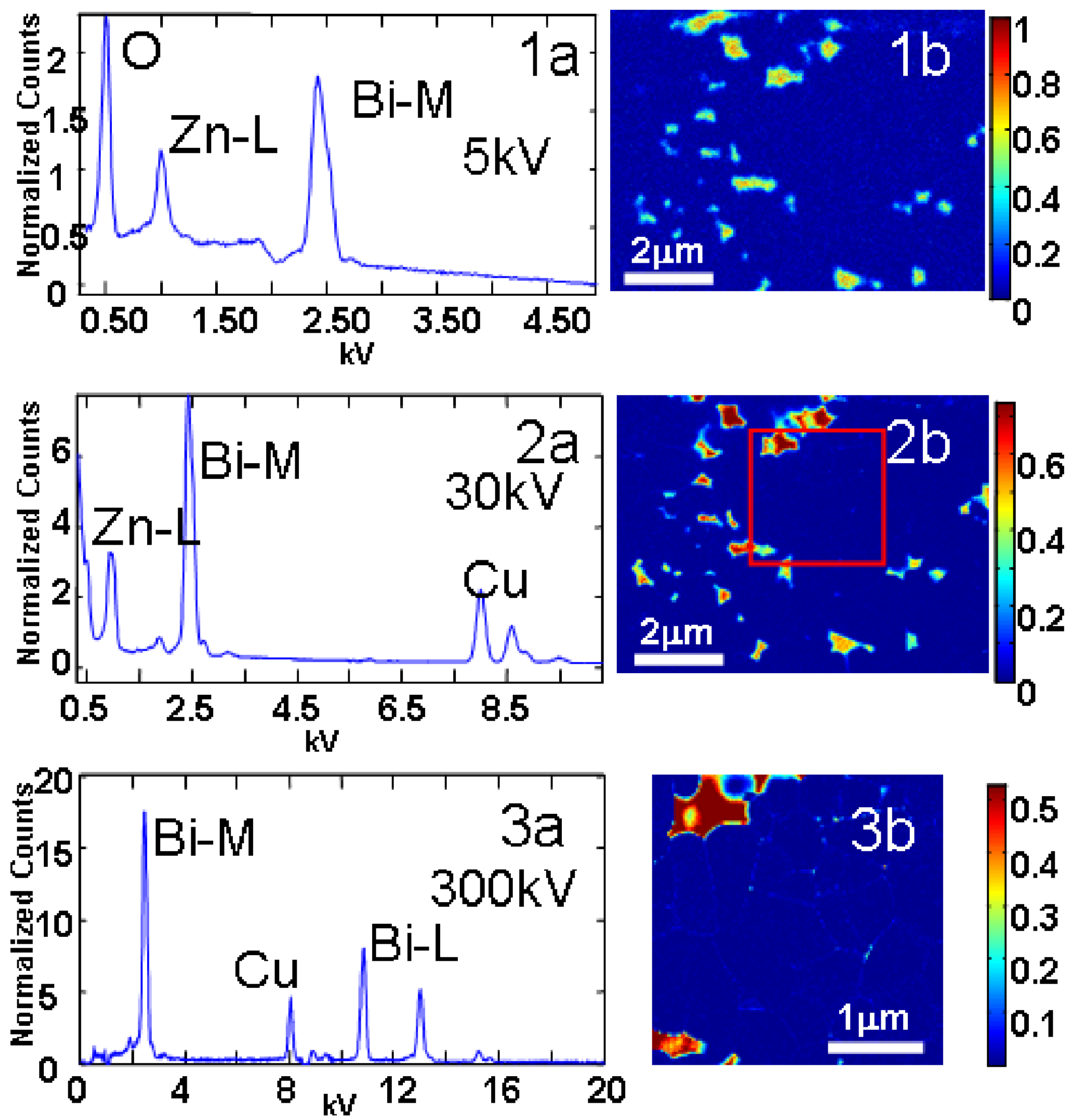

FIG. 1a and b. Bi component spectrum and image respectively from the Roentec Si-drift detector with the SEM operated at $5 \mathrm{kV}$. FIG. $2 \mathrm{a}$ and $\mathrm{b}$. Bi component spectrum and image respectively from the Roentec Si-drift detector with the SEM operated at $30 \mathrm{kV}$. The red box shows the common area between FIG1 and 2 and FIG. 3. FIG 3a and b. Bi component spectrum and image from analysis at $300 \mathrm{kV}$ in a TEM/STEM with $\mathrm{Si}(\mathrm{Li})$ detector. 